\title{
On the optimal paving over MASAs in von Neumann algebras
}

\author{
By Sorin Popa 1 And StefaAn Vaes 2 \\ In Operator Algebras and Their Applications: A Tribute to Richard V. Kadison, \\ Contemporary Mathematics 671 (2016), pp. 199-208.
}

\begin{abstract}
We prove that if $A$ is a singular MASA in a $\mathrm{II}_{1}$ factor $M$ and $\omega$ is a free ultrafilter, then for any $x \in M \ominus A$, with $\|x\| \leq 1$, and any $n \geq 2$, there exists a partition of 1 with projections $p_{1}, p_{2}, \ldots, p_{n} \in A^{\omega}$ (i.e. a paving) such that $\left\|\sum_{i=1}^{n} p_{i} x p_{i}\right\| \leq 2 \sqrt{n-1} / n$, and give examples where this is sharp. Some open problems on optimal pavings are discussed.
\end{abstract}

\section{Introduction}

A famous problem formulated by R.V. Kadison and I.M. Singer in 1959 asked whether the diagonal MASA (maximal abelian ${ }^{*}$-subalgebra) $\mathcal{D}$ of the algebra $\mathcal{B}\left(\ell^{2} \mathbb{N}\right)$, of all linear bounded operators on the Hilbert space $\ell^{2} \mathbb{N}$, satisfies the paving property, requiring that for any contraction $x=x^{*} \in \mathcal{B}\left(\ell^{2} \mathbb{N}\right)$ with 0 on the diagonal, and any $\varepsilon>0$, there exists a partition of 1 with projections $p_{1}, \ldots, p_{n} \in \mathcal{D}$, such that $\left\|\sum_{i} p_{i} x p_{i}\right\| \leq \varepsilon$. This problem has been settled in the affirmative by A. Marcus, D. Spielman and N. Srivastava in [MSS13], with an actual estimate $n \leq 12^{4} \varepsilon^{-4}$ for the paving size, i.e., for the minimal number $n=n(x, \varepsilon)$ of such projections.

In a recent paper [PV14, we considered a notion of paving for an arbitrary MASA in a von Neumann algebra $A \subset M$, that we called so-paving, which requires that for any $x=x^{*} \in M$ and any $\varepsilon>0$, there exist $n \geq 1$, a net of partitions of 1 with $n$ projections $p_{1, i}, \ldots, p_{n, i} \in A$ and projections $q_{i} \in M$ such that $\left\|q_{i}\left(\sum_{k=1}^{n} p_{k, i} x p_{k, i}-a_{i}\right) q_{i}\right\| \leq \varepsilon, \forall i$, and $q_{i} \rightarrow 1$ in the so-topology.

This property is in general weaker than the classic Kadison-Singer norm paving, but it coincides with it for the diagonal MASA $\mathcal{D} \subset \mathcal{B}\left(\ell^{2} \mathbb{N}\right)$. We conjectured in [PV14] that any MASA $A \subset M$ satisfies so-paving. We used the results in [MSS13] to check this conjecture for all MASAs in type I von Neumann algebras, and all Cartan MASAs in amenable von Neumann algebras and in group measure space factors arising from profinite actions, with the estimate $12^{4} \varepsilon^{-4}$ for the so-paving size derived from MSS13] as well.

We also showed in PV14] that if $A$ is the range of a normal conditional expectation, $E: M \rightarrow A$, and $\omega$ is a free ultrafilter on $\mathbb{N}$, then so-paving for $A \subset M$ is equivalent to the usual KadisonSinger paving for the ultrapower MASA $A^{\omega} \subset M^{\omega}$, with the norm paving size for $A^{\omega} \subset M^{\omega}$ coinciding with the so-paving size for $A \subset M$. In the case $A$ is a singular MASA in a $\mathrm{II}_{1}$ factor $M$, norm-paving for the ultrapower inclusion $A^{\omega} \subset M^{\omega}$ has been established in [P13], with paving size $1250 \varepsilon^{-3}$. This estimate was improved to $<16 \varepsilon^{-2}+1$ in [PV14], while also shown to be $\geq \varepsilon^{-2}$ for arbitrary MASAs in $\mathrm{II}_{1}$ factors.

\footnotetext{
${ }^{1}$ Mathematics Department, UCLA, CA 90095-1555 (United States), popa@math.ucla.edu Supported in part by NSF Grant DMS-1401718

${ }^{2} \mathrm{KU}$ Leuven, Department of Mathematics, Leuven (Belgium), stefaan.vaes@wis.kuleuven.be Supported by ERC Consolidator Grant 614195 from the European Research Council under the European Union's Seventh Framework Programme.
} 
In this paper we prove that the paving size for singular MASAs in $\mathrm{II}_{1}$ factors is in fact $<4 \varepsilon^{-2}+1$, and that for certain singular MASAs this is sharp. More precisely, we prove that for any contraction $x \in M^{\omega}$ with 0 expectation onto $A^{\omega}$, and for any $n \geq 2$, there exists a partition of 1 with $n$ projections $p_{i} \in A^{\omega}$ such that $\left\|\sum_{i=1}^{n} p_{i} x p_{i}\right\| \leq 2 \sqrt{n-1} / n$. In fact, given any finite set of contractions $F \subset M^{\omega} \ominus A^{\omega}$, we can find a partition $p_{1}, \ldots, p_{n} \in A^{\omega}$ that satisfies this estimate for all $x \in F$, so even the multipaving size for singular MASAs is $<4 \varepsilon^{-2}+1$.

To construct pavings satisfying this estimate, we first use Theorem 4.1(a) in P13] to get a unitary $u \in A^{\omega}$ with $u^{n}=1, \tau\left(u^{k}\right)=0,1 \leq k \leq n-1$, such that any word with alternating letters from $\left\{u^{k} \mid 1 \leq k \leq n-1\right\}$ and $F \cup F^{*}$ has trace 0 . This implies that for each $x \in F$ the set $X=\left\{u^{i-1} x u^{-i+1} \mid i=1,2, \ldots, n\right\}$ satisfies the conditions $\tau\left(\Pi_{k=1}^{m}\left(x_{2 k-1} x_{2 k}^{*}\right)\right)=0=$ $\tau\left(\Pi_{k=1}^{m}\left(x_{2 k-1}^{*} x_{2 k}\right)\right)$, for all $m$ and all $x_{k} \in X$ with $x_{k} \neq x_{k+1}$ for all $k$. We call $L$-freeness this property of a subset of a $\mathrm{II}_{1}$ factor. We then prove the general result, of independent interest, that any L-free set of contractions $\left\{x_{1}, \ldots, x_{n}\right\}$ satisfies the norm estimate $\left\|\sum_{i=1}^{n} x_{i}\right\| \leq 2 \sqrt{n-1}$. We do this by first "dilating" $\left\{x_{1}, \ldots, x_{n}\right\}$ to an L-free set of unitaries $\left\{U_{1}, \ldots, U_{n}\right\}$ in a larger $\mathrm{II}_{1}$ factor, for which we deduce the Kesten-type estimate $\left\|\sum_{i=1}^{n} U_{i}\right\|=2 \sqrt{n-1}$ from results in AO74. This implies the inequality for the L-free contractions as well. By applying this to $\left\{u^{i-1} x u^{1-i} \mid i=1, \ldots, n\right\}$ and taking into account that $\frac{1}{n} \sum_{i=1}^{n} u^{i-1} x u^{1-i}=\sum_{i=1}^{n} p_{i} x p_{i}$, where $p_{1}, \ldots, p_{n}$ are the minimal spectral projections of $u$, we get $\left\|\sum_{i=1}^{n} p_{i} x p_{i}\right\| \leq 2 \sqrt{n-1} / n, \forall x \in F$.

We also notice that if $M$ is a $\mathrm{II}_{1}$ factor, $A \subset M$ is a MASA and $v \in M$ a self-adjoint unitary of trace 0 which is free with respect to $A$, then $\left\|\sum_{i=1}^{n} p_{i} v p_{i}\right\| \geq 2 \sqrt{n-1} / n$ for any partition of 1 with projections in $A^{\omega}$, with equality if and only if $\tau\left(p_{i}\right)=1 / n, \forall i$. A concrete example is when $M=L(\mathbb{Z} *(\mathbb{Z} / 2 \mathbb{Z})$ ), $A=L(\mathbb{Z})$ (which is a singular MASA in $M$ by [P81]) and $v=v^{*} \in L(\mathbb{Z} / 2 \mathbb{Z}) \subset M$ denotes the canonical generator. This shows that the estimate $4 \varepsilon^{-2}+1$ for the paving size is in this case optimal.

The constant $2 \sqrt{n-1}$ is known to coincide with the spectral radius of the $n$-regular tree, and with the first eigenvalue less than $n$ of $n$-regular Ramanujan graphs. Its occurence in this context leads us to a more refined version of a conjecture formulated in PV14, predicting that for any MASA $A \subset M$ which is range of a normal conditional expectation, any $n \geq 2$ and any contraction $x=x^{*} \in M$ with 0 expectation onto $A$, the infimum $\varepsilon(A \subset M ; n, x)$ over all norms of pavings of $x,\left\|\Sigma_{i=1}^{n} p_{i} x p_{i}\right\|$, with $n$ projections $p_{1}, \ldots, p_{n}$ in $A^{\omega}, \Sigma_{i} p_{i}=1$, is bounded above by $2 \sqrt{n-1} / n$, and that in fact $\sup \left\{\varepsilon(A \subset M ; n, x) \mid x=x^{*} \in M \ominus A,\|x\| \leq 1\right\}=2 \sqrt{n-1} / n$. Such an optimal estimate would be particularly interesting to establish for the diagonal MASA $\mathcal{D} \subset \mathcal{B}\left(\ell^{2} \mathbb{Z}\right)$.

\section{Preliminaries}

A well known result of $\mathrm{H}$. Kesten in [K58] shows that if $\mathbb{F}_{k}$ denotes the free group with $k$ generators $h_{1}, \ldots, h_{k}$, and $\lambda$ is the left regular representation of $\mathbb{F}_{k}$ on $\ell^{2} \mathbb{F}_{k}$, then the norm of the Laplacian operator $L=\sum_{i=1}^{k}\left(\lambda\left(h_{i}\right)+\lambda\left(h_{i}^{-1}\right)\right)$ is equal to $2 \sqrt{2 k-1}$. It was also shown in [K58] that, conversely, if $k$ elements $h_{1}, \ldots, h_{k}$ in a group $\Gamma$ satisfy $\left\|\sum_{i=1}^{k} \lambda\left(h_{i}\right)+\lambda\left(h_{i}^{-1}\right)\right\|=2 \sqrt{2 k-1}$, then $h_{1}, \ldots, h_{k}$ are freely independent, generating a copy of $\mathbb{F}_{k}$ inside $\Gamma$. The calculation of the norm of $L$ in K58 uses the formalism of random walks on groups, but it really amounts to calculating the higher moments $\tau\left(L^{2 n}\right)$ and using the formula $\|L\|=\lim _{m}\left(\tau\left(L^{2 m}\right)\right)^{1 / 2 m}$, where $\tau$ denotes the canonical (normal faithful) tracial state on the group von Neumann algebra $L\left(\mathbb{F}_{k}\right)$.

Kesten's result implies that whenever $u_{1}, \ldots, u_{k}$ are freely independent Haar unitaries in a type $\mathrm{II}_{1}$ factor $M$ (i.e., $u_{1}, \ldots, u_{k}$ generate a copy of $L\left(\mathbb{F}_{k}\right)$ inside $\left.M\right)$, then one has $\left\|\sum_{i=1}^{k} u_{i}+u_{i}^{*}\right\|=$ 
$2 \sqrt{2 k-1}$. In particular, if $M$ is the free group factor $L\left(\mathbb{F}_{k}\right)$ and $u_{i}=\lambda\left(h_{i}\right)$, where $h_{1}, \ldots, h_{k} \in \mathbb{F}_{k}$ as above, then $\left\|\Sigma_{i=1}^{k} \alpha_{i} u_{i}+\overline{\alpha_{i}} u_{i}^{*}\right\|=2 \sqrt{2 k-1}$, for any scalars $\alpha_{i} \in \mathbb{C}$ with $\left|\alpha_{i}\right|=1$.

Estimates of norms of linear combinations of elements satisfying more general free independence relations in group $\mathrm{II}_{1}$ factors $L(\Gamma)$ have later been obtained in [L73], [B74], [AO74] $]^{3}$ These estimates involve elements in $L(\Gamma)$ (viewed as convolvers on $\ell^{2} \Gamma$ ) that are supported on a subset $\left\{g_{1}, \ldots, g_{n}\right\} \subset \Gamma$ satisfying the following weaker freeness condition, introduced in [L73]: whenever $k \geq 1$ and $i_{s} \neq j_{s}, j_{s} \neq i_{s+1}$ for all $s$, we have that

$$
g_{i_{1}} g_{j_{1}}^{-1} \cdots g_{i_{k}} g_{j_{k}}^{-1} \neq e .
$$

In [B74] and AO74], this is called the Leinert property and it is proved to be equivalent with $\left\{g_{1}^{-1} g_{2}, \ldots, g_{1}^{-1} g_{n}\right\}$ freely generating a copy of $\mathbb{F}_{n-1}$. The most general calculation of norms of elements $x=\Sigma_{i} c_{i} \lambda\left(g_{i}\right) \in L(\Gamma)$, supported on a Leinert set $\left\{g_{i}\right\}_{i}$, with arbitrary coefficients $c_{i} \in \mathbb{C}$, was obtained by Akemann and Ostrand in AO74. The calculation shows in particular that if $\left\{g_{1}, \ldots, g_{n}\right\}$ satisfies Leinert's freeness condition then $\left\|\sum_{i=1}^{n} \lambda\left(g_{i}\right)\right\|=2 \sqrt{n-1}$. Since $h_{1}, \ldots, h_{k} \in \Gamma$ freely independent implies $\left\{h_{i}, h_{i}^{-1} \mid 1 \leq i \leq k\right\}$ is a Leinert set, the result in AO74 does recover Kesten's theorem as well. Like in [K58], the norm of an element of the form $L=\sum_{i=1}^{n} c_{i} \lambda\left(g_{i}\right)$ in $\mathrm{AO} 74$ is calculated by evaluating $\lim _{n} \tau\left(\left(L^{*} L\right)^{n}\right)^{1 / 2 n}$ (by computing the generating function of the moments of $\left.L^{*} L\right)$.

An argument similar to [K58] was used in [Le96] to prove that, conversely, if some elements $g_{1}, \ldots, g_{n}$ in a group $\Gamma$ satisfy $\left\|\sum_{i=1}^{n} \lambda\left(g_{i}\right)\right\|=2 \sqrt{n-1}$, then $g_{1}, \ldots, g_{n}$ is a Leinert set. On the other hand, note that if $g_{1}, \ldots, g_{n}$ are $n$ arbitrary elements in an arbitrary group $\Gamma$ and we denote $L=\sum_{i=1}^{n} \lambda\left(g_{i}\right)$ the corresponding Laplacian, then the $n$ 'th moment $\tau\left(\left(L^{*} L\right)^{n}\right)$ is bounded from below by the $n$ 'th moment of the Laplacian obtained by taking $g_{i}$ to be the generators of $\mathbb{F}_{n}$. Thus, we always have $\left\|\sum_{i=1}^{n} \lambda\left(g_{i}\right)\right\| \geq 2 \sqrt{n-1}$. More generally, if $v_{1}, \ldots, v_{n}$ are unitaries in a von Neumann algebra $M$ with normal faithful trace state $\tau$, such that any word $v_{i_{1}} v_{j_{1}}^{*} v_{i_{2}} v_{j_{2}}^{*} \ldots v_{i_{m}} v_{j_{m}}^{*}, \forall m \geq 1, \forall 1 \leq i_{k}, j_{k} \leq n$, has trace with non-negative real part, then $\left\|\sum_{i=1}^{n} v_{i}\right\| \geq 2 \sqrt{n-1}$. In particular, for any unitaries $u_{1}, \ldots, u_{n} \in M$ one has $\left\|\sum_{i=1}^{n} u_{i} \otimes \overline{u_{i}}\right\| \geq$ $2 \sqrt{n-1}$.

For convenience, we state below some norm calculations from [AO74, formulated in the form that will be used in the sequel:

Proposition 2.1 ([AO74] $)$. If $v_{1}, v_{2}, \ldots, v_{n-1} \in M$ are freely independent Haar unitaries, then

$$
\left\|1+\Sigma_{i=1}^{n-1} v_{i}\right\|=2 \sqrt{n-1} .
$$

Also, if $\alpha_{0}, \ldots, \alpha_{n-1} \in \mathbb{C}, \Sigma_{i}\left|\alpha_{i}\right|^{2}=1$, then

$$
\left\|\alpha_{0} 1+\sum_{i=1}^{n-1} \alpha_{i} v_{i}\right\| \leq 2 \sqrt{1-1 / n} .
$$

Note that (2.1) above shows in particular that if $p, q \in M$ are projections with $\tau(p)=1 / 2$ and $\tau(q)=1 / n$, for some $n \geq 3$, and they are freely independent, then $\|q p q\|=1 / 2+$ $\sqrt{n-1} / n$. Indeed, any two such projections can be thought of as embedded into $L\left(\mathbb{F}_{2}\right)$ with $p$ and $q$ lying in the MASAs of the two generators, $p \in A_{1}$, respectively $q \in A_{2}$. Denote $v=2 p-1$. Let $q_{1}=q, q_{2}, \ldots, q_{n} \in A_{2}$ be mutually orthogonal projections of trace $1 / n$ and denote $u=\sum_{j=1}^{n} \lambda^{j-1} q_{j}$, where $\lambda=2 \exp (2 \pi i / n)$. It is then easy to see that the elements

\footnotetext{
${ }^{3}$ See also the more "rough" norm estimates for elements in $L\left(\mathbb{F}_{n}\right)$ obtained by R. Powers in 1967 in relation to another problem of Kadison, but published several years later in [Po75], and which motivated in part the work in $\mathrm{AO} 74$.
} 
$v_{k}=v u^{k} v u^{-k}, k=1,2, \ldots, n-1$ are freely independent Haar unitaries. By (2.1) we thus have $\left\|\sum_{k=0}^{n-1} u^{k} v u^{-k}\right\|=\left\|1+\sum_{k=1}^{n-1} v u^{k} v u^{-k}\right\|=2 \sqrt{n-1}$. But $\sum_{k=0}^{n-1} u^{k} v u^{-k}=n\left(\sum_{j=1}^{n} q_{j} v q_{j}\right)$, implying that

$$
\|q v q\|=\|q(2 p-1) q\|=2 \sqrt{n-1} / n=2 \sqrt{\tau(q)(1-\tau(q))}
$$

or equivalently

$$
\|q p q\|=1 / 2+\sqrt{n-1} / n=\tau(p)+\sqrt{\tau(q)(1-\tau(q))} .
$$

The computation of the norm of the product of freely independent projections $q, p$ of arbitrary trace in $M$ (in fact, of the whole spectral distribution of $q p q$ ) was obtained by Voiculescu in [Vo86], as one of the first applications of his multiplicative free convolution (which later became a powerful tool in free probability). We recall here these norm estimates, which in particular show that the first of the above norm calculations holds true for projections $q$ of arbitrary trace (see also [ABH87] for the case $\tau(q)=1 / n, \tau(p)=1 / m$, for integers $n \geq m \geq 2$ ):

Proposition 2.2 ([Vo86]). If $p, q \in M$ are freely independent projections with $\tau(q) \leq \tau(p) \leq$ $1 / 2$, then

$$
\|q p q\|=\tau(p)+\tau(q)-2 \tau(p) \tau(q)+2 \sqrt{\tau(p) \tau(1-p) \tau(q) \tau(1-q)} .
$$

If in addition $\tau(p)=1 / 2$ and we denote $v=2 p-1$, then

$$
\|q v q\|=2 \sqrt{\tau(q) \tau(1-q)} .
$$

\section{$3 \quad L$-free sets of contractions and their dilation}

Recall from [P13] that two selfadjoint sets $X, Y \subset M \ominus \mathbb{C} 1$ of a tracial von Neumann algebra $M$ are called freely independent set $\S^{4}$ if the trace of any word with letters alternating from $X$ and $Y$ is equal to 0 . Also, a subalgebra $B \subset M$ is called freely independent of a set $X$, if $X$ and $B \ominus \mathbb{C} 1$ are freely independent as sets. Several results were obtained in [P13] about constructing a "large subalgebra" $B$ inside a given subalgebra $Q \subset M$ that is freely independent of a given countable set $X$. Motivated by a condition appearing in one such result, namely [P13, Theorem 4.1], and by a terminology used in [AO74, we consider in this paper the following free independence condition for arbitrary elements in tracial algebras:

Definition 3.1. Let $(M, \tau)$ be a von Neumann algebra with a normal faithful tracial state. A subset $X \subset M$ is called $L$-free ${ }^{5}$ if

$$
\tau\left(x_{1} x_{2}^{*} \cdots x_{2 k-1} x_{2 k}^{*}\right)=0 \text { and } \tau\left(x_{1}^{*} x_{2} \cdots x_{2 k-1}^{*} x_{2 k}\right)=0,
$$

whenever $k \geq 1, x_{1}, \ldots, x_{2 k} \in X$ and $x_{i} \neq x_{i+1}$ for all $i=1, \ldots, 2 k-1$.

Note that if the subset $X$ in the above definition is taken to be contained in the set of canonical unitaries $\left\{u_{g} \mid g \in \Gamma\right\}$ of a group von Neumann algebra $M=L(\Gamma)$, i.e. $X=\left\{u_{g} \mid g \in F\right\}$ for some subset $F \subset \Gamma$, then L-freeness of $X$ amounts to $F$ being a Leinert set. But the key

\footnotetext{
${ }^{4}$ We specifically consider this condition for subsets $X, Y \subset M \ominus \mathbb{C} 1$, not to be confused with the freeness of the von Neumann algebras generated by $X$ and $Y$.

${ }^{5}$ Note that this notion is not the same as (and should not be confused with) the notion of L-sets used in Pi92.
} 
example of an L-free set that is important for us here occurs from a diffuse algebra $B$ that is free independent from a set $Y=Y^{*} \subset M \ominus \mathbb{C} 1$ : given any $y_{1}, \ldots, y_{n} \in Y$ and any unitary element $u \in \mathcal{U}(B)$ with $\tau\left(u^{k}\right)=0,1 \leq k \leq n-1$, the set $\left\{u^{k-1} y_{k} u^{-k+1} \mid 1 \leq k \leq n\right\}$ is L-free.

Note that we do need to impose both conditions on the traces being zero in Definition 3.1, because we cannot deduce $\tau\left(x_{1}^{*} x_{2} x_{3}^{*} x_{1}\right)=0$ from $\tau\left(y_{1} y_{2}^{*} y_{3} y_{4}^{*}\right)=0$ for all $y_{i} \in X$ with $y_{1} \neq y_{2}$, $y_{2} \neq y_{3}, y_{3} \neq y_{4}$. However, if $X \subset \mathcal{U}(M)$ consists of unitaries, then only one set of conditions is sufficient. We in fact have:

Lemma 3.2. Let $X=\left\{u_{1}, \ldots, u_{n}\right\} \subset \mathcal{U}(M)$. Then the following conditions are equivalent

(a) $X$ is an L-free set.

(b) $\tau\left(u_{i_{1}} u_{j_{1}}^{*} \cdots u_{i_{k}} u_{j_{k}}^{*}\right)=0$ whenever $k \geq 1$ and $i_{s} \neq j_{s}, j_{s} \neq i_{s+1}$ for all $s$.

(c) $u_{1}^{*} u_{2}, \ldots, u_{1}^{*} u_{n}$ are free generators of a copy of $L\left(\mathbb{F}_{n-1}\right)$.

Proof. This is a trivial verification.

Corollary 3.3. If $\left\{u_{1}, \ldots, u_{n}\right\}$ is an L-free set of unitaries in $\mathcal{U}(M)$, then $\left\|\sum_{i=1}^{n} u_{i}\right\|=2 \sqrt{n-1}$. Moreover, if $\alpha_{1}, \ldots, \alpha_{n} \in \mathbb{C}$ with $\sum_{i=1}^{n}\left|\alpha_{i}\right|^{2} \leq 1$, then

$$
\left\|\sum_{i=1}^{n} \alpha_{i} u_{i}\right\| \leq 2 \sqrt{1-1 / n} .
$$

Proof. Since $\left\|\sum_{i=1}^{n} \alpha_{i} u_{i}\right\|=\left\|\alpha_{1} 1+\sum_{i=2}^{n} \alpha_{i} u_{1}^{*} u_{i}\right\|$, the statement follows by applying (2.2) to the freely independent Haar unitaries $v_{j}=u_{1}^{*} u_{j}, 2 \leq j \leq n$.

Proposition 3.4. Let $M$ be a finite von Neumann algebra with a faithful tracial state $\tau$. If $\left\{x_{1}, \ldots, x_{n}\right\} \subset M$ is an $L$-free set with $\left\|x_{i}\right\| \leq 1$ for all $i$, then there exists a tracial von Neumann algebra $(\mathcal{M}, \tau)$, a trace preserving unital embedding $M \subset \mathcal{M}$ and an L-free set of unitaries $\left\{U_{1}, \ldots, U_{n}\right\} \subset \mathcal{U}(\widetilde{\mathcal{M}})$ with $\widetilde{\mathcal{M}}=M_{n+1}(\mathbb{C}) \otimes \mathcal{M}$ so that, denoting by $\left(e_{i j}\right)_{i, j=0, \ldots, n}$ the matrix units of $M_{n+1}(\mathbb{C})$, we have $e_{00} U_{i} e_{00}=x_{i}$ for all $i$.

Proof. Define $\mathcal{M}=M * L\left(\mathbb{F}_{n(n-1)}\right)$ and denote by $u_{i, j}, i \neq j$, free generators of $L\left(\mathbb{F}_{n(n-1)}\right)$. For every $i \in\{1, \ldots, n\}$, define

$$
c_{i}=\sqrt{1-x_{i} x_{i}^{*}} \text { and } d_{i}=-\sqrt{1-x_{i}^{*} x_{i}} .
$$

Put $\widetilde{\mathcal{M}}=M_{n+1}(\mathbb{C}) \otimes \mathcal{M}$ and define the unitary elements $U_{i} \in \mathcal{U}(\widetilde{\mathcal{M}})$ given by

$$
U_{i}=\left(e_{00} \otimes x_{i}\right)+\left(e_{i i} \otimes x_{i}^{*}\right)+\left(e_{0 i} \otimes c_{i}\right)+\left(e_{i 0} \otimes d_{i}\right)+\sum_{j \neq i}\left(e_{j j} \otimes u_{i, j}\right) .
$$

Note that $U_{i}$ is the direct sum of the unitary

$$
\left(\begin{array}{cc}
x_{i} & c_{i} \\
d_{i} & x_{i}^{*}
\end{array}\right) \text { in positions } 0 \text { and } i \text {, and the unitary } \bigoplus_{j \neq i} u_{i, j} \text { in the positions } j \neq i \text {. }
$$

By construction, we have that $e_{00} U_{i} e_{00}=x_{i} e_{00}$. So, it remains to prove that $\left\{U_{1}, \ldots, U_{n}\right\}$ is L-free.

Take $k \geq 1$ and indices $i_{s}, j_{s}$ such that $i_{s} \neq j_{s}, j_{s} \neq i_{s+1}$ for all $s$. We must prove that

$$
\tau\left(U_{i_{1}} U_{j_{1}}^{*} \cdots U_{i_{k}} U_{j_{k}}^{*}\right)=0
$$


Consider $V:=U_{i_{1}} U_{j_{1}}^{*} \cdots U_{i_{k}} U_{j_{k}}^{*}$ as a matrix with entries in $\mathcal{M}$. Every entry of this matrix is a sum of "words" with letters

$$
\left\{x_{i}, x_{i}^{*}, c_{i}, d_{i} \mid i=1, \ldots, n\right\} \cup\left\{u_{i, j}, u_{i, j}^{*} \mid i \neq j\right\} .
$$

We prove that every word that appears in a diagonal entry $V_{i i}$ of $V$ has zero trace. The following types of words appear.

$1^{\circ}$ Words without any of the letters $u_{a, b}$ or $u_{a, b}^{*}$. These words only appear as follows:

- in the entry $V_{00}$ as $x_{i_{1}} x_{j_{1}}^{*} \cdots x_{i_{k}} x_{j_{k}}^{*}$, which has zero trace;

- if $i_{1}=j_{k}=i$, in the entry $V_{i i}$ as $w=d_{i} x_{j_{1}}^{*} x_{i_{2}} x_{j_{2}}^{*} \cdots x_{i_{k-1}} x_{j_{k-1}}^{*} x_{i_{k}} d_{i}^{*}$. Then we have

$$
\begin{aligned}
\tau(w) & =\tau\left(x_{j_{1}}^{*} x_{i_{2}} \cdots x_{j_{k-1}}^{*} x_{i_{k}} d_{i}^{*} d_{i}\right) \\
& =\tau\left(x_{j_{1}}^{*} x_{i_{2}} \cdots x_{j_{k-1}}^{*} x_{i_{k}}\right)-\tau\left(x_{j_{1}}^{*} x_{i_{2}} \cdots x_{j_{k-1}}^{*} x_{i_{k}} x_{i}^{*} x_{i}\right) \\
& =0-\tau\left(x_{i_{1}} x_{j_{1}}^{*} \cdots x_{i_{k}} x_{j_{k}}^{*}\right)=0
\end{aligned}
$$

because $i=i_{1}$ and $i=j_{k}$.

$2^{\circ}$ Words with exactly one letter of the type $u_{a, b}$ or $u_{a, b}^{*}$. These words have zero trace because $\tau\left(M u_{a, b} M\right)=\{0\}$.

$3^{\circ}$ Words $w$ with two or more letters of the type $u_{a, b}$ or $u_{a, b}^{*}$. Consider two consecutive such letters in $w$, i.e. a subword of $w$ of the form

$$
u_{i, j}^{\varepsilon} w_{0} u_{i^{\prime}, j^{\prime}}^{\varepsilon^{\prime}}
$$

with $\varepsilon, \varepsilon^{\prime}= \pm 1$ and where $w_{0}$ is a word with letters from $\left\{x_{i}, x_{i}^{*}, c_{i}, d_{i} \mid i=1, \ldots, n\right\}$. We distinguish three cases.

- $\left(\varepsilon^{\prime}, i^{\prime}, j^{\prime}\right) \neq(-\varepsilon, i, j)$.

- $u_{i, j} w_{0} u_{i, j}^{*}$.

- $u_{i, j}^{*} w_{0} u_{i, j}$.

To prove that $\tau(w)=0$, it suffices to prove that in the last two cases, we have that $\tau\left(w_{0}\right)=0$.

A subword of the form $u_{i, j} w_{0} u_{i, j}^{*}$ can only arise from the $j j$-entry of

$$
U_{i_{s}} U_{j_{s}}^{*} \cdots U_{i_{t}} U_{j_{t}}^{*} \quad \text { with } i_{s}=j_{t}=i, j_{s}=i_{t}=j
$$

(and thus, $t \geq s+2$ ). In that case,

$$
w_{0}=c_{j}^{*} x_{i_{s+1}} x_{j_{s+1}}^{*} \cdots x_{i_{t-1}} x_{j_{t-1}}^{*} c_{j} .
$$

Thus,

$$
\begin{aligned}
\tau\left(w_{0}\right) & =\tau\left(x_{i_{s+1}} x_{j_{s+1}}^{*} \cdots x_{i_{t-1}} x_{j_{t-1}}^{*} c_{j} c_{j}^{*}\right) \\
& =\tau\left(x_{i_{s+1}} x_{j_{s+1}}^{*} \cdots x_{i_{t-1}} x_{j_{t-1}}^{*}\right)-\tau\left(x_{i_{s+1}} x_{j_{s+1}}^{*} \cdots x_{i_{t-1}} x_{j_{t-1}}^{*} x_{j} x_{j}^{*}\right) \\
& =0-\tau\left(x_{j_{s}} x_{i_{s+1}}^{*} \cdots x_{j_{t-1}}^{*} x_{i_{t}}\right)=0
\end{aligned}
$$

because $j=j_{s}$ and $j=i_{t}$. 
Finally, a subword of the form $u_{i, j}^{*} w_{0} u_{i, j}$ can only arise from the $j j$-entry of

$$
U_{j_{s-1}}^{*} U_{i_{s}} \cdots U_{j_{t-1}}^{*} U_{i_{t}} \quad \text { with } j_{s-1}=i_{t}=i, i_{s}=j_{t-1}=j
$$

(and thus, $t \geq s+2$ ). In that case,

$$
w_{0}=d_{j} x_{j_{s}}^{*} x_{i_{s+1}} \cdots x_{j_{t-2}}^{*} x_{i_{t-1}} d_{j}^{*} .
$$

As above, it follows that $\tau\left(w_{0}\right)=0$.

So, we have proved that every word that appears in a diagonal entry $V_{i i}$ of $V$ has trace zero. Then also $\tau(V)=0$ and it follows that $\left\{U_{1}, \ldots, U_{n}\right\}$ is an L-free set of unitaries.

Corollary 3.5. Let $(M, \tau)$ be a finite von Neumann algebra with a faithful normal tracial state. If $\left\{x_{1}, \ldots, x_{n}\right\} \subset M$ is L-free with $\left\|x_{i}\right\| \leq 1$ for all $i$, then

$$
\left\|\sum_{i=1}^{n} x_{i}\right\| \leq 2 \sqrt{n-1}
$$

More generally, given any complex scalars $\alpha_{1}, \ldots, \alpha_{n}$ with $\sum_{i=1}^{n}\left|\alpha_{i}\right|^{2} \leq 1$, we have

$$
\left\|\sum_{i=1}^{n} \alpha_{i} x_{i}\right\| \leq 2 \sqrt{1-1 / n} .
$$

Proof. Assuming $n \geq 2$, with the notations from Proposition 3.4 and by using Corollary 3.3 . we have $\left\|\sum_{i=1}^{n} \alpha_{i} U_{i}\right\| \leq 2 \sqrt{1-1 / n}$. Reducing with the projection $e_{00}$, it follows that

$$
\left\|\sum_{i=1}^{n} \alpha_{i} x_{i}\right\| \leq 2 \sqrt{1-1 / n}
$$

\section{Applications to paving problems}

Like in [P13, PV14], if $\mathcal{A} \subset \mathcal{M}$ is a MASA in a von Neumann algebra and $x \in \mathcal{M}$, then we denote by $\mathrm{n}(\mathcal{A} \subset \mathcal{M} ; x, \varepsilon)$ the smallest $n$ for which there exist projections $p_{1}, \ldots, p_{n} \in \mathcal{A}$ and $a \in \mathcal{A}$ such that $\|a\| \leq\|x\|, \sum_{i=1}^{n} p_{i}=1$ and $\left\|\sum_{i=1}^{n} p_{i} x p_{i}-a\right\| \leq \varepsilon\|x\|$ (with the convention that $\mathrm{n}(\mathcal{A} \subset \mathcal{M} ; x, \varepsilon)=\infty$ if no such finite partition exists), and call it the paving size of $x$.

Recall also from [D54] that a MASA $\mathcal{A}$ in a von Neumann algebra $\mathcal{M}$ is called singular, if the only unitary elements in $\mathcal{M}$ that normalize $\mathcal{A}$ are the unitaries in $\mathcal{A}$.

Theorem 4.1. Let $A_{n} \subset M_{n}$ be a sequence of singular MASAs in finite von Neumann algebras and $\omega$ a free ultrafilter on $\mathbb{N}$. Denote $\mathbf{M}=\prod_{\omega} M_{n}$ and $\mathbf{A}=\prod_{\omega} A_{n}$. Given any countable set of contractions $X \subset \mathbf{M} \ominus \mathbf{A}$ and any integer $n \geq 2$, there exists a partition of 1 with projections $p_{1}, \ldots, p_{n} \in \mathbf{A}$ such that

$$
\left\|\sum_{j=1}^{n} p_{j} x p_{j}\right\| \leq 2 \sqrt{n-1} / n, \quad \text { for all } x \in X .
$$

In particular, the paving size of $\mathbf{A} \subset \mathbf{M}$,

$$
\mathrm{n}(\mathbf{A} \subset \mathbf{M} ; \varepsilon) \stackrel{\text { def }}{=} \sup \left\{\mathrm{n}(\mathbf{A} \subset \mathbf{M} ; x, \varepsilon) \mid x=x^{*} \in \mathbf{M} \ominus \mathbf{A}\right\},
$$

is less than $4 \varepsilon^{-2}+1$, for any $\varepsilon>0$. 
Proof. By Theorem 4.1(a) in P13, there exists a diffuse abelian von Neumann subalgebra $A_{0} \subset \mathbf{A}$ such that for any $k \geq 1$, any word with alternating letters $x=x_{0} \Pi_{i=1}^{k}\left(v_{i} x_{i}\right)$ with $x_{i} \in X, 1 \leq i \leq k-1, x_{0}, x_{k} \in X \cup\{1\}, v_{i} \in A_{0} \ominus \mathbb{C} 1$, has trace equal to 0 .

This implies that if $p_{1}, \ldots, p_{n} \in \mathbf{A}$ are projections of trace $1 / n$ summing up to 1 and we denote $u=\sum_{j=1}^{n} \lambda^{j-1} p_{j}$, where $\lambda=\exp (2 \pi i / n)$, then for any $x \in X$ the set $\left\{u^{i-1} x u^{-i+1}\right.$ $i=1,2, \ldots, n\}$ is L-free. Since $\frac{1}{n} \sum_{i=1}^{n} u^{i-1} x u^{1-i}=\sum_{i=1}^{n} p_{i} x p_{i}$, where $p_{1}, \ldots, p_{n}$ are the minimal spectral projections of $u$, by Proposition 3.4 it follows that for all $x \in X$ we have

$$
\left\|\sum_{i=1}^{n} p_{i} x p_{i}\right\|=\frac{1}{n}\left\|\sum_{i=1}^{n} u^{i-1} x u^{-i+1}\right\| \leq 2 \sqrt{n-1} / n .
$$

To derive the last part, let $\varepsilon>0$ and denote by $n$ the integer with the property that $2 n^{-1 / 2} \leq$ $\varepsilon<2(n-1)^{-1 / 2}$. If $x \in \mathbf{M} \ominus \mathbf{A},\|x\| \leq 1$, and $p_{1}, \ldots, p_{n} \in \mathbf{A}$ are mutually orthogonal projections of trace $1 / n$ that satisfy the free independence relation with $X=\{x\}$ as above, then $n<4 \varepsilon^{-2}+1$ and we have

$$
\left\|\sum_{i=1}^{n} p_{i} x p_{i}\right\| \leq 2 \sqrt{n-1} / n \leq \varepsilon,
$$

showing that $\mathrm{n}(\mathbf{A} \subset \mathbf{M} ; x, \varepsilon)<4 \varepsilon^{-2}+1$.

Remark 4.2. The above result suggests that an alternative way of measuring the so-paving size over a MASA in a von Neumann algebra $A \subset M$ admitting a normal conditional expectation, is by considering the quantity

$$
\varepsilon(A \subset M ; n) \stackrel{\text { def }}{=} \sup _{x \in\left(M_{h}^{\omega} \ominus A^{\omega}\right)_{1}}\left(\inf \left\{\left\|\Sigma_{i=1}^{n} p_{i} x p_{i}\right\| \mid p_{i} \in \mathcal{P}\left(A^{\omega}\right), \Sigma_{i} p_{i}=1\right\}\right) .
$$

With this notation, the above theorem shows that for a singular MASA in a $\mathrm{II}_{1}$ factor $A \subset M$, one has $\varepsilon(A \subset M ; n) \leq 2 \sqrt{n-1} / n, \forall n \geq 2$, a formulation that's slightly more precise than the estimate $\mathrm{n}_{\mathrm{s}}(A \subset M ; \varepsilon)=\mathrm{n}\left(A^{\omega} \subset M^{\omega} ; \varepsilon\right)<4 \varepsilon^{-2}+1$. Also, the conjecture $\left(2.8 .2^{\circ}\right.$ in [PV14]) about the so-paving size can this way be made more precise, by asking whether $\varepsilon(A \subset$ $M ; n) \leq 2 \sqrt{n-1} / n, \forall n$, for any MASA with a normal conditional expectation $A \subset M$. It seems particularly interesting to study this question in the classical Kadison-Singer case of the diagonal MASA $\mathcal{D} \subset \mathcal{B}=\mathcal{B}\left(\ell^{2} \mathbb{N}\right)$, and more generally for Cartan MASAs $A \subset M$. So far, the solution to the Kadison-Singer paving problem in [MSS13. shows that $\varepsilon(\mathcal{D} \subset \mathcal{B} ; n) \leq 12 n^{-1 / 4}$.

Also, while by CEKP07] one has $\mathrm{n}(\mathcal{D} \subset \mathcal{B} ; \varepsilon) \geq \varepsilon^{-2}$ and by [PV14] one has $\mathrm{n}_{\mathrm{s}}(A \subset M ; \varepsilon)=$ $\mathrm{n}\left(A^{\omega} \subset M^{\omega} ; \varepsilon\right) \geq \varepsilon^{-2}$, for any MASA in a $\mathrm{II}_{1}$ factor $A \subset M$, it would be interesting to decide whether $\varepsilon(\mathcal{D} \subset \mathcal{B} ; n)$ and $\varepsilon(A \subset M ; n)$ are in fact bounded from below by $2 \sqrt{n-1} / n, \forall n$.

For a singular MASA in a $\mathrm{II}_{1}$ factor, $A \subset M$, combining 4.1 with such a lower bound would show that $\varepsilon(A \subset M ; n)=2 \sqrt{n-1} / n, \forall n$. While we could not prove this general fact, let us note here that for certain singular MASAs this equality holds indeed.

Proposition 4.3. $1^{\circ}$ Let $M$ be a $\mathrm{II}_{1}$ factor and $A \subset M$ a MASA. Assume $v \in M$ is a unitary element with $\tau(v)=0$ such that $A$ is freely independent of the set $\left\{v, v^{*}\right\}$ (i.e., any alternating word in $A \ominus \mathbb{C} 1$ and $\left\{v, v^{*}\right\}$ has trace 0$)$. Then for any partition of 1 with projections $p_{1}, \ldots, p_{n} \in A^{\omega}$ we have $\left\|\sum_{i=1}^{n} p_{i} v p_{i}\right\| \geq 2 \sqrt{n-1} / n$, with equality iff all $p_{i}$ have trace $1 / n$. Also, $\varepsilon(A \subset M ; n) \geq 2 \sqrt{n-1} / n, \forall n$.

$2^{\circ}$ If $M=L(\mathbb{Z} *(\mathbb{Z} / 2 \mathbb{Z})), A=L(\mathbb{Z})$ and $v=v^{*}$ denotes the canonical generator of $L(\mathbb{Z} / 2 \mathbb{Z})$, then $\varepsilon(A \subset M ; v, n)=\varepsilon(A \subset M ; n)=2 \sqrt{n-1}, \forall n$. 
Proof. The free independence assumption in $1^{\circ}$ implies that $A^{\omega} \ominus \mathbb{C}$ and $\left\{v, v^{*}\right\}$ are freely independent sets as well. This in turn implies that for each $i$, the projections $p_{i}$ and $v p_{i} v^{*}$ are freely independent, and so by Proposition 2.2 one has $\left\|p_{i} v p_{i}\right\|=\left\|p_{i} v p_{i} v^{*}\right\|=2 \sqrt{\tau\left(p_{i}\right)\left(1-\tau\left(p_{i}\right)\right)}$. Thus, if one of the projections $p_{i}$ has trace $\tau\left(p_{i}\right)>1 / n$, then $\left\|\Sigma_{j} p_{j} v p_{j}\right\| \geq\left\|p_{i} v p_{i}\right\|>2 \sqrt{n-1} / n$, while if $\tau\left(p_{i}\right)=1 / n, \forall i$, then $\left\|\Sigma_{j} p_{j} v p_{j}\right\|=2 \sqrt{n-1} / n$.

By applying $1^{\circ}$ to part $2^{\circ}$, then using 4.1 and the fact that $A=L(\mathbb{Z})$ is singular in $M=$ $L(\mathbb{Z} *(\mathbb{Z} / 2 \mathbb{Z}))($ cf. $[\mathrm{P} 81])$, proves the last part of the statement.

\section{References}

[AO74] C.A. Akemann and P.A. Ostrand, Computing norms in group C*-algebras. Amer. J. Math. 98 (1976), 1015-1047.

[ABH87] J. Anderson, B. Blackadar and U. Haagerup, Minimal projections in the reduced group $C^{*}$-algebra of $\mathbb{Z}_{n} * \mathbb{Z}_{m}$, J. Operator Theory 26 (1991), 3-23.

[B74] M. Bozejko, On $\Lambda(p)$ sets with minimal constant in discrete noncommutative groups. Proc. Amer. Math. Soc. 51 (1975), 407-412.

[CEKP07] P. Casazza, D. Edidin, D. Kalra and V.I. Paulsen, Projections and the Kadison-Singer problem. Oper. Matrices 1 (2007), 391-408.

[D54] J. Dixmier, Sous-anneaux abéliens maximaux dans les facteurs de type fini, Ann. of Math. 59 (1954), 279-286.

[KS59] R.V. Kadison and I.M. Singer, Extensions of pure states, Amer. J. Math. 81 (1959), 383-400.

[K58] H. Kesten, Symmetric random walks on groups. Trans. Amer. Math. Soc. 92 (1959), 336354.

[Le96] F. Lehner, A characterization of the Leinert property. Proc. Amer. Math. Soc. 125 (1997), 3423-3431.

[L73] M. Leinert, Faltungsoperatoren auf gewissen diskreten Gruppen. Studia Math. 52 (1974), 149-158.

[MSS13] A.W. Marcus, D.A. Spielman and N. Srivastava, Interlacing families II: mixed characteristic polynomials and the Kadison-Singer problem. Ann. of Math. 182 (2015), 327-350.

[Pi92] G. Pisier, Multipliers and lacunary sets in non-amenable groups, American J. Math. 117 (1995), 337-376.

[P81] S. Popa, Orthogonal pairs of *-subalgebras in finite von Neumann algebras, J. Operator Theory, 9 (1983), 253-268.

[P13] S. Popa, A II 1 factor approach to the Kadison-Singer problem. Comm. Math. Phys. 332 (2014), 379-414.

[PV14] S. Popa and S. Vaes, Paving over arbitrary MASAs in von Neumann algebra, to appear in Analysis and PDE. arXiv:1412.0631

[Po75] R. Powers: Simplicity of the $\mathrm{C}^{*}$-algebra associated with the free group on two generators, Duke Mathematical Journal 42 (1975), 151-156.

[Vo86] D. Voiculescu, Multiplication of certain noncommuting random variables. J. Operator Theory 18 (1987), 223-235. 